\title{
SYSTÈMES LINÉAIRES SUR LES OPÉRATEURS DE MIKUSIŃSKI ET COMMANDE D'UNE POUTRE FLEXIBLE
}

\author{
MICHEL FLIESS, HUGUES MOUNIER, PIERRE ROUCHON, AND JOACHIM \\ RUDOLPH
}

\begin{abstract}
An algebraic framework is proposed for linear systems over Mikusiński operators. It is illustrated by the control synthesis of an Euler-Bernoulli flexible beam.
\end{abstract}

\section{Résumé}

On propose une théorie algébrique des systèmes linéaires sur les opérateurs de Mikusiński, illustrée par la commande d'une poutre flexible d'Euler-Bernoulli.

Mots-clés : systèmes linéaires, anneaux, modules, commandabilité, observabilité, matrices de transfert, opérateurs de Mikusiński, poutres flexibles, équation aux dérivées partielles d'Euler-Bernoulli, conjecture de Serre, fonctions de Gevrey-Roumieu.

Classification mathématique: Primaire 93C20, 73K05, 44A40;

Secondaire 13C10, 26E10.

\section{INTRODUCTION}

Cette communication, qui fait suite à $[25,38,40]$, où étaient examinés quelques cas concrets de commande associés à l'équation des cordes vibrantes, traite d'une poutre flexible d'Euler-Bernoulli, qui, sous des formes variées, a déjà suscité une abondante littérature (voir, par exemple, [2, 9 , $10,30,52]$ ). On cherche à réduire la flexion et, donc, les vibrations d'une telle poutre en mouvement.

Nous posons les linéaments d'une théorie algébrique des systèmes linéaires constants, qui, généralisant $[16,17,19,21,23,38]$, se traduit par la considération de modules de type fini sur des anneaux commutatifs et intègres. On y dégage les notions de commandabilité, d'observabilité et de matrices de transfert. Pour aborder des équations comme celle d'Euler-Bernoulli ${ }^{1}$, l'anneau de base est engendré par des opérateurs de Mikusiński [33, 34], ou, plus précisément, des fonctions opérationnelles [33, 34] ; rappelons que le calcul opérationnel de Mikusiński [33, 34] (voir, aussi, [13, 53]) fournit un substitut aussi élégant que maniable de la transformation de Laplace. La liberté d'un certain module, dont les propriétés s'obtiennent par des arguments de nature homologique $(c f .[14,29])^{2}$, permet, en assignant une trajectoire à une base de ce module, d'obtenir le comportement désiré de la poutre avec une commande en boucle ouverte : c'est une démarche analogue à celle de la platitude [22] en non-linéaire de dimension finie. Une régulation avec passivité, proche de la boucle proportionnelle-dérivée utilisée en [1],

\footnotetext{
${ }^{1}$ Le lien entre équations aux dérivées partielles linéaires et modules est, dans un esprit tout différent, déjà connu à travers la théorie des $D$-modules (voir, par exemple, [48]).

${ }^{2}$ Nous faisons notamment appel à la résolution de la conjecture de Serre par Quillen [42] et Suslin [49], déjà exploitée en [38] (voir, aussi, [23, 24]).
} 
conduit à la stabilisation. Les calculs numériques emploient les développements en série des opérateurs de Mikusiński [34] et un résultat dû à Ramis [43] sur les fonctions de type Gevrey-Roumieu.

Il n'est pas question, dans ce cadre restreint, de comparer notre approche qui, en son stade actuel, ne s'applique qu'aux équations aux dérivées partielles linéaires, à coefficients constants et en une seule variable d'espace, aux remarquables développements théoriques dus au Professeur J.-L. Lions (voir, par exemple, $[31,32]$ ) et à son école ( $c f .[3,27])$, dont on connaît l'importance pour les structures flexibles $(c f .[28])^{3}$. Ajoutons, toutefois, que ce parallèle avec d'autres points de vue $(c f .[3,6,11])$ a été mené à bien dans la thèse récente de l'un des auteurs [38] (voir, aussi, [23, 24, 37, 39]) à propos d'une autre famille en dimension infinie, les systèmes linéaires à retards purs.

\section{Systèmes $A$-LinÉaires}

\subsection{Généralités}

Les notions de commandabilité, d'observabilité et de matrices de transfert introduites ci-dessous sont adaptées de [16, 19, 23, 24, 38]. Quant au langage algébrique, il est élémentaire et se trouve, sauf mention contraire, dans bien des manuels, comme, par exemple, [14, 29].

Soit $A$ un anneau commutatif, intègre, avec élément unité. Un système $A$-linéaire $(\Sigma)$ est un $A$-module $\Lambda$ de type fini. Notons $[\xi]$ le sous- $A$-module engendré par une partie $\xi$ de $\Lambda$. Une entrée $\boldsymbol{u}=\left(u_{1}, \ldots, u_{m}\right)$ est une partie finie de $\Lambda$, telle que le module quotient $\Lambda /[\boldsymbol{u}]$ soit de torsion; $\boldsymbol{u}$ est dite indépendante si, et seulement si, $[\boldsymbol{u}]$ est un $A$-module libre de rang $m$. Une sortie $\boldsymbol{y}=\left(y_{1}, \ldots, y_{p}\right)$ est une partie finie de $\Lambda$.

Soit $B$ une $A$-algèbre. Le système ( $\Sigma$ ) est dit $B$-commandable sans torsion (resp. projectif, libre) si, et seulement si, le $B$-module $B \otimes_{A} \Lambda$, obtenu par extension de l'anneau de base, est sans torsion (resp. projectif, libre). La $B$-commandabilité libre (resp. projective) implique la $B$-commandabilité projective (resp. sans torsion).

Le résultat suivant découle de [46] :

Théorème et définition 1 . Supposons $(\Sigma) A$-commandable sans torsion. Soit $\mathcal{S}$ une partie multiplicative de $A$ telle que $\mathcal{S}^{-1} A$ soit un anneau de Bezout ${ }^{4}$. Alors il existe un élément $\pi \in \mathcal{S}, \pi \neq 0$, tel que le $A\left[\pi^{-1}\right]$-module localisé $\Lambda_{\pi}=A\left[\pi^{-1}\right] \otimes_{A} \Lambda$ soit libre. Le système $(\Sigma)$ est dit $\pi$-libre.

Le système $(\Sigma)$, muni d'une entrée $\boldsymbol{u}$ et d'une sortie $\boldsymbol{y}$, est dit $B$-observable si, et seulement si, les deux $B$-modules $B \otimes_{A} \Lambda$ et $B \otimes_{A}[\boldsymbol{u}, \boldsymbol{y}]$ coïncident.

Soit $K$ le corps de fractions de $A$. Le foncteur de Laplace $\Lambda \rightarrow \tilde{\Lambda}=$ $K \otimes{ }_{A} \Lambda, \lambda \mapsto \tilde{\lambda}=1 \otimes \lambda$ entre $A$-modules et $K$-espaces vectoriels associe à tout élément $\lambda \in \Lambda$ sa transformée de Laplace formelle $\tilde{\lambda} \in \tilde{\Lambda}$. Soit un système d'entrée $\boldsymbol{u}$ indépendante et de sortie $\boldsymbol{y}$. Alors, $\tilde{\boldsymbol{u}}=\left(\tilde{u}_{1}, \ldots, \tilde{u}_{m}\right)$ est une base de $\tilde{\Lambda}$. La matrice $T$, à coefficients dans $K$, telle que $\tilde{\boldsymbol{y}}=T \tilde{\boldsymbol{u}}$, est la matrice de transfert de $(\Sigma)$.

\footnotetext{
${ }^{3}$ Renvoyons, aussi, à $[4,8]$ pour la considération d'équations voisines des nôtres.

${ }^{4}$ Un anneau $R$ est de Bezout si tout idéal finiment engendré de $R$ est principal ( $c f$. $[45])$.
} 


\subsection{EXemples}

Exemple 1. Prenons pour $A$ l'anneau principal $k\left[\frac{d}{d t}\right]$, où $k$ est un corps commutatif, $\mathbb{R}$ par exemple. On obtient ainsi les systèmes linéaires constants de dimension finie ( $c f$. $[16,17,19]$ ), où, comme dans l'approche comportementale (en américain, behavioral) de Willems [51], il n'est pas nécessaire de distinguer les variables. Rappelons que la commandabilité à la Kalman se traduit alors par la liberté du module correspondant.

Exemple 2. Posons $A=\mathbb{R}\left[\frac{d}{d t}, \delta_{1}, \ldots, \delta_{r}\right]$, oì $\delta_{i}, i=1, \ldots, r$, est un opérateur de retard pur : pour toute fonction $f: \mathbb{R} \rightarrow \mathbb{R}, \delta_{i} f(t)=f\left(t-h_{i}\right)$, $h_{i} \in \mathbb{R}_{+}$. On obtient ainsi les systèmes linéaires constants à retards purs (voir [38] et, aussi, [23, 24]).

Exemple 3. Avec $A=k[\delta]$, où $\delta$ est un opérateur de retard pur, on obtient (cf. [18]) les systèmes linéaires constants en temps discret, de dimension finie.

\subsection{Remarques}

Remarque 4. Au contraire de [23, 38], nous ne chercherons pas à donner, comme en $[16,21]$, une représentation d'état de $(\Sigma)$. Elle semble, en effet, inutile pour les applications en vue.

REMARQUE 5. Les anneaux non commutatifs apparaissent naturellement en bien des circonstances. Citons, ici, les $D$-modules (cf. [48]), qui formalisent certaines questions sur les opérateurs différentiels linéaires à coefficients variables, et les systèmes linéaires instationnaires de dimension finie (voir $[16,19])$.

Remarque 6. Notre formalisme est bien adapté à des questions comme la dualité [47] ou l'interconnexion [20].

\section{Opérateurs de Mikusiński}

\subsection{BREF RAPPEL}

Les fonctions continues $[0,+\infty[\rightarrow \mathbb{C}$ forment pour l'addition et le produit de convolution un anneau commutatif, qui, d'après un théorème fameux de Titchmarsh, est intègre. Son corps de fractions $\mathcal{M}$ est l'ensemble des opérateurs de Mikusiński [33, 34] (voir, aussi, [13, 53]). L'élément unité de $\mathcal{M}$ est l'opérateur de Dirac. L'opérateur de dérivation est noté s.

\subsection{Systèmes de Mikusiński}

Un système linéaire $(\Sigma)$ sur les opérateurs de Mikusiński, ou, plus brièvement, un système de Mikusiński, est un système $A$-linéaire, où $A \subset \mathcal{M}$ est une $k$-algèbre de type fini, $k$ étant un sous-corps de $\mathbb{C}$.

Exemple 7 . Avec $A=k\left[s, e^{-h_{1} s}, \ldots, e^{-h_{r} s}\right], h_{1}, \ldots, h_{r} \in \mathbb{R}_{+}$, où $e^{-h_{1} s}, \ldots$, $e^{-h_{r} s}$ sont des opérateurs de translation $(c f .[33,34])$, on retrouve les systèmes linéaires à retards purs.

Exemple 8. Avec $A$ engendrée par $s$ et un nombre fini d'opérateurs de Mikusiński, on obtient les systèmes linéaires à retards purs ou répartis quelconques.

Exemple 9. Avec $A=k\left[s^{\alpha}\right], \alpha \in \mathbb{C}$, on obtient (cf. [21]) les systèmes linéaires à dérivation non entière, popularisés en automatique et théorie du signal par Oustaloup [41]. 


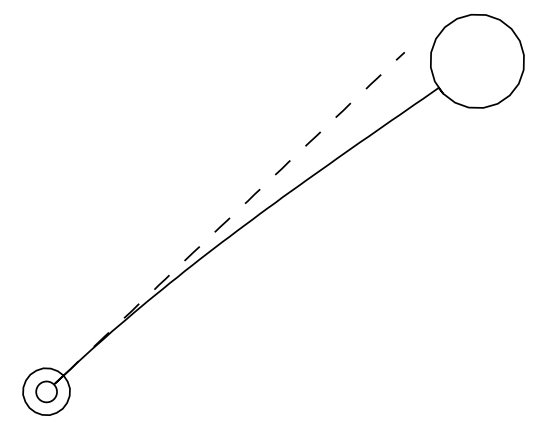

Figure 1. poutre d'Euler-Bernoulli en rotation avec une masse au bout.

\subsection{Systèmes paramétriques de Mikusiński}

On considère l'anneau $R_{I}$ des fonctions opérationnelles $(c f .[33,34])$ $I \rightarrow \mathcal{M}$, où $I$ est un intervalle de $\mathbb{R}$. Un système linéaire paramétrique sur les opérateurs de Mikusinski, ou, plus brièvement, un système paramétrique de Mikusiński est un module de type fini sur une $k$-algèbre $A \subset R_{I}$ intègre et de type fini. Un exemple naturel est donné ci-dessous.

\section{LA POUTRE D'Euler-Bernoulli}

\subsection{LE MODÈLE}

Le mouvement d'une poutre flexible de longueur $R$, dont l'extrémité $x=0$ est encastrée dans l'axe d'un moteur d'angle $\theta$ et dont l'autre extrémité $x=R$ est solidaire d'une masse $m$ ponctuelle, est supposé obéir à l'équation d'Euler-Bernoulli (élasticité linéaire, flexion faible, inertie de la poutre négligeable par rapport à la masse $m$, forces de Coriolis négligeables, c'est-à-dire $\dot{\theta}$ petit) et aux conditions aux bords suivantes :

$$
\begin{aligned}
\frac{\partial^{2} w}{\partial \tau^{2}} & =-\frac{\partial^{4} w}{\partial^{4} x} \\
w(0, \tau) & =0, \quad \frac{\partial w(0, \tau)}{\partial x}=0 \\
\frac{\partial^{2} w(1, \tau)}{\partial x^{2}} & =0, \quad \frac{\partial^{3} w(1, \tau)}{\partial x^{3}}=k_{1} \frac{d^{2}}{d \tau^{2}} \theta(\tau)+k_{2} \frac{\partial^{2} w(1, \tau)}{\partial \tau^{2}}
\end{aligned}
$$

où $w(x, t)$ est le champ des déformations de la poutre par rapport à l'axe tournant d'angle $\theta$ et où $\frac{d^{2} \theta}{d \tau^{2}}$ est la commande. Les égalités suivantes définissent le temps $t=R^{2} \sqrt{\rho S /(E I)} \tau$, la longueur $r=R x$ et les deux paramètres $k_{1}=m /(\rho S)$ et $k_{2}=k_{1} / R$ où $E, I, \rho$ et $S$ sont des grandeurs physiques usuelles.

Remarque 10. Nous renvoyons à [5] pour la modélisation de manipulateurs flexibles.

\subsection{Modules}

Avec les conditions initiales $w(x, 0)=0, \frac{\partial w(x, 0)}{\partial \tau}=0$, le calcul opérationnel de Mikusiński [33, 34] associe à (1a) l'équation différentielle ordinaire, dépendant de $s, s^{2} \hat{w}=-\hat{w}^{(4)}$ : la notation $\hat{w}$ dénote la fonction opérationnelle correspondant à $w$. Comme $\sqrt{s}$ et $i \sqrt{s}$ sont des opérateurs logarithmiques 
$[33,34]$, il vient, en posant $\xi=\exp (i \pi / 4)$,

$$
\hat{w}(x, s)=a e^{x \dot{\xi} \sqrt{s}}+b e^{-x \xi \sqrt{s}}+c e^{x \bar{\xi} \sqrt{s}}+d e^{-x \bar{\xi} \sqrt{s}}
$$

où $a, b, c, d$ découlent des conditions aux bords :

$$
\begin{aligned}
& a+b+c+d=0 \\
& \xi(a-b)+\bar{\xi}(c-d)=0 \\
& a \frac{\exp (\xi \sqrt{s})}{\sqrt{s}}+b \frac{\exp (-\xi \sqrt{s})}{\sqrt{s}}-c \frac{\exp (\bar{\xi} \sqrt{s})}{\sqrt{s}}-d \frac{\exp (-\bar{\xi} \sqrt{s})}{\sqrt{s}}=0 \\
& a \xi \frac{\exp (\xi \sqrt{s})}{\sqrt{s}}-b \xi \frac{\exp (-\xi \sqrt{s})}{\sqrt{s}}-c \bar{\xi} \frac{\exp (\bar{\xi} \sqrt{s})}{\sqrt{s}}+d \bar{\xi} \frac{\exp (-\bar{\xi} \sqrt{s})}{\sqrt{s}}=-i \hat{u}
\end{aligned}
$$

La variable $\hat{u}$ correspond à $k_{1} \theta(\tau)+k_{2} w(1, \tau)$ et, moyennant un bouclage élémentaire, joue le rôle d'entrée.

Posons $A=\mathbb{C}\left[\frac{e^{ \pm \xi \sqrt{s}}}{\sqrt{s}}, \frac{e^{ \pm \bar{\xi} \sqrt{s}}}{\sqrt{s}}\right]$. Soit $M$ le $A$-module engendré par $a, b, c, d$ et $\hat{u}$.

Lemme 11. $M$ est de rang 1 , sans torsion, mais non libre.

Démonstration. Les deux premières assertions sont faciles. Pour la troisième, considérons la matrice de présentation de $M$ suivante

$$
\left(\begin{array}{ccccc}
1 & 1 & 1 & 1 & 0 \\
\xi & -\xi & \bar{\xi} & -\bar{\xi} & 0 \\
z_{1} & z_{1}^{-1} & -z_{2} & -z_{2}^{-1} & 0 \\
\xi z_{1} & -\xi z_{1}^{-1} & -\bar{\xi} z_{2} & \bar{\xi} z_{2}^{-1} & i
\end{array}\right)
$$

où $z_{1}=\frac{e^{\xi \sqrt{s}}}{\sqrt{s}}, z_{2}=\frac{e^{\bar{\xi} \sqrt{s}}}{\sqrt{s}}$. Les mineurs d'ordre 4 sont nuls pour $z_{1}=-z_{2}=1$. D'après un lemme de [7] et la résolution de la conjecture de Serre en [42, 49], $M$ n'est pas libre.

Posons $e=(c+d) / 2, f=(c-d) / 2$. Alors,

$$
e(\cosh (\xi \sqrt{s})+\cosh (\bar{\xi} \sqrt{s})) / \sqrt{s}=f(i \sinh (\xi \sqrt{s})-\sinh (\bar{\xi} \sqrt{s})) / \sqrt{s}
$$

Il vient :

Lemme 12. Le module $A\left[\pi^{-1}\right] \otimes_{A} M$, où $\pi=\frac{i \sinh (\xi \sqrt{s})-\sinh (\bar{\xi} \sqrt{s})}{\xi s \sqrt{s}}$, est
libre, de base $y=\pi^{-1} e$.

Notons que

$$
\hat{u}=-2(2+\cosh (\sqrt{2 s})+\cosh (i \sqrt{2 s})) y
$$

et

$$
\begin{aligned}
& \hat{w}(x, s)=((\cosh (\xi \sqrt{s})+\cosh (\bar{\xi} \sqrt{s}))(i \sinh (x \xi \sqrt{s})+\sinh (x \bar{\xi} \sqrt{s})) \\
& \quad+(i \sinh (\xi \sqrt{s})-\sinh (\bar{\xi} \sqrt{s}))(-\cosh (x \xi \sqrt{s})+\cosh (x \bar{\xi} \sqrt{s})) \frac{2 \sqrt{s}}{\xi} y
\end{aligned}
$$

Résumons (3) et (4) par $\hat{u}=\gamma y, \gamma \in \mathcal{M}$ et $\hat{w}=\delta y$, ò̀ $\delta$ est une fonction opérationnelle. Il vient :

ThÉORÈme 13. Le $\mathbb{C}[\gamma, \delta]$-module $\Lambda$ engendré par $\hat{u}$ et $\hat{w}$ est sans torsion, de rang 1. Le $\mathbb{C}\left[\gamma, \delta, \gamma^{-1}, \delta^{-1}\right]$-module localisé $\Lambda_{\gamma, \delta}$, engendré par $\hat{u}$ et $\hat{w}$, est libre, de base $y$.

En d'autres termes, il y a $\mathbb{C}[\gamma, \delta]$-commandabilité sans torsion (resp. $\mathbb{C}[\gamma$, $\left.\delta, \gamma^{-1}, \delta^{-1}\right]$-commandabilité libre) pour le $\mathbb{C}[\gamma, \delta]$-système (resp. $\mathbb{C}[\gamma, \delta$, $\left.\gamma^{-1}, \delta^{-1}\right]$-système) paramétrique de Mikusiński, associé à $\Lambda$ (resp. $\left.\Lambda_{\gamma, \delta}\right)$. 


\subsection{Remarques}

Remarque 14. Par rapport à la platitude [22], déjà évoquée en introduction, il existe une grande labilité de choix du module et, donc éventuellement, d'une base. Cela est dî à l'appartenance des opérateurs de Mikusiński à un corps, $\mathcal{M}$. La sélection doit reposer sur des critères de commodité de calcul (série convergente) et de sens physique.

REMARQUe 15. Un sujet connu d'investigation est la nature et l'emplacement des actionneurs et, aussi, des capteurs (voir, par exemple, [15]). Soit une poutre commandée par un actionneur piézoélectrique (cf. [12]) sur un intervalle. Une démarche analogue à ce qui précède conduit à une équation d'Euler-Bernoulli pour chacune des trois parties de la poutre et à des conditions de bords et de raccords appropriées : le calcul sur les modules en découle. Un travail ultérieur développera ce point de vue tout en le comparant à d'autres approches (cf. [50]).

\subsection{Calcul de la trajectoire et de la commande en boucle OUVERTE}

Le développement des formules (3) et (4) fait intervenir des séries convergentes d'opérateurs du type $\sum_{n>0} \alpha_{n} s^{n}$. Une fonction $C^{\infty} f: I \rightarrow \mathbb{R}$, où $I$ est un intervalle ouvert de $\mathbb{R}$, est de classe $\mathcal{C}\{\Gamma(\mu n)\}$ (cf. [34]), si, et seulement si, il existe $M_{f}, A_{f} \in \mathbb{R}_{+}$tels que, pour tout $t \in I$ et pour tout ordre de dérivation $n$,

$$
\left|f^{(n)}(t)\right| \leq M_{f} \Gamma(\mu n)\left(A_{f}\right)^{n}
$$

où $\Gamma$ est la fonction d'Euler. Autrement dit, les fonctions de classe $\mathcal{C}\{\Gamma(\mu n)\}$ sont les fonctions régulières $f$ telles que la série $\sum_{n>0} f^{(n)}(t) X^{n}$ soit Gevrey d'ordre $\mu(c f .[43])^{5}$ uniformément en $t$. Il est clair que les fonctions analytiques a ppartiennent à $\mathcal{C}\{\Gamma(\mu n)\}$, où $\mu \geq 1$. Pour $\mu>1$, la classe $\mathcal{C}\{\Gamma(\mu n)\}$ est, d'après un théorème dû à Denjoy-Carleman ( $c f$. [34]), bien plus vaste : elle comporte, en particulier, des fonctions "plateau" du type partition de l'unité ( $c f$. [34, exemple de la page 125 , avec $\left.\varepsilon_{n}=(\Gamma(\mu n))^{-1 / n}\right]$ ou la fonction $y(\tau)$ ci-dessous). Enfin, l'ensemble des fonctions de classe $\mathcal{C}\{\Gamma(\mu n)\}$ forme un anneau pour l'addition et le produit usuel. Ce type d'espace de fonctions est aussi considéré en [26] sous le nom d'espaces de type $S$.

Si $y(\tau)$ est de classe $\mathcal{C}\{\Gamma(\mu n)\}, \mu<2$, la série suivante, qui correspond à $\cosh (\sqrt{2 s}) y$, est absolument convergente

$$
\sum_{n \geq 0} 2^{n} y^{(n)}(\tau) /(2 n) !
$$

Pour toute spécialisation de la base $y$ en une fonction de classe $\mathcal{C}\{\Gamma(\mu n)\}$, $\mu<2$, on obtient, pour (3),

$$
u(\tau)=-4\left(y(\tau)+\sum_{n \geq 0} 2^{2 n} y^{(2 n)}(\tau)\right) /(4 n) !
$$

et pour (4),

$$
\begin{aligned}
w(x, \tau)=4 \sum_{n \geq 0} \frac{(-1)^{n+1}}{(4 n+3) !}\left[(x-1)^{4 n+3}+\right. & \Re\left((x+i)^{4 n+3}\right)- \\
& \left.\Im\left((x+i)^{4 n+3}\right)\right] y^{(2 n+2)}(\tau),
\end{aligned}
$$

\footnotetext{
${ }^{5}$ Renvoyons à [44] pour un excellent rappel sur les séries divergentes.
} 


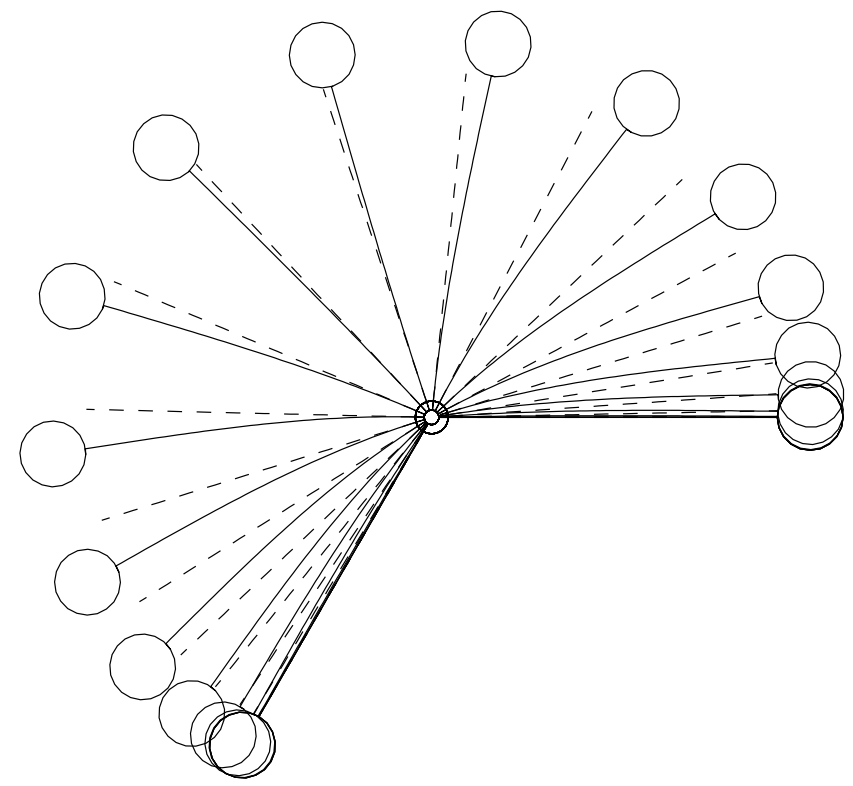

Figure 2. les diverses positions de la poutre au cours du temps.

où $\Re$ (resp. $\Im$ ) indique la partie réelle (resp. imaginaire). En d'autres termes, les deux relations ci-dessus donnent une famille, paramétrée par toutes les fonctions de classe $\mathcal{C}\{\Gamma(\mu n)\}, \mu<2$, de trajectoires pour le système hybride (1a).

Considérons maintenant deux états d'équilibre de la poutre correspondant aux deux angles $\theta_{1}$ et $\theta_{2}$. Les formules ci-dessus permettent de construire, pour tout temps $T>0$, grâce à une fonction plateau $[0, T] \ni \tau \mapsto y(\tau)$ de classe $\mathcal{C}\{\Gamma(\mu n)\}$ avec $y(0)=-k_{1} \theta_{1} / 8, y(T)=-k_{1} \theta_{2} / 8$ et pour tout $n>0, y^{(n)}(0)=y^{(n)}(T)=0$, une commande régulière $[0, T] \ni \tau \mapsto \theta(\tau)=$ $\left(u(\tau)-k_{2} w(1, \tau)\right) / k_{1}$. Cette commande correspond à une rotation d'angle $\theta_{2}-\theta_{1}$ pendant le temps $T$. Elle garantit l'absence de vibration en fin de mouvement $\tau \geq T$. Les calculs des figures 2,3 et 4 correspondent à une poutre d'aluminium de $24 \mathrm{~g}$, de $0.575 \mathrm{~m}$ de long et d'une masse de $m=250 \mathrm{~g}\left(E I=0.0603 \mathrm{~N} \mathrm{~m}^{2}, \rho S=0.0423 \mathrm{~kg} \mathrm{~m}^{-1}\right)$. Le mouvement de $4 \pi / 3 \mathrm{rad}$ s'effectue en $10 \mathrm{~s}\left(\theta_{1}=0\right.$ et $\left.\theta_{2}=4 \pi / 3\right)$ avec

$$
y(\tau)=-\frac{k_{1} \theta_{2}}{8} \frac{\int_{0}^{\tau / T} \exp \left(-(p(1-p))^{-10 / 9}\right) d p}{\int_{0}^{1} \exp \left(-(p(1-p))^{-10 / 9}\right) d p} .
$$

En utilisant la proposition 5 de [43] caractérisant les fonctions GevreyRoumieu de type $s>0$, il est facile de montrer que la fonction

$$
] 0,1\left[\ni t \mapsto \exp \left(-\left(t(1-t)^{-\nu}\right)\right)\right.
$$

$\nu>0$, est de classe $\mathcal{C}\{\Gamma(\mu n)\}, \mu=1+1 / \nu$. La fonction $y$ ci-dessus est, donc, de classe $\mathcal{C}\{\Gamma((\mu n)\}$, avec $\mu=1.9<2$. Les séries sont calculées avec une trentaine de termes.

\subsection{Bouclage stabilisant}

Pour suivre avec stabilité asymptotique les trajectoires en boucle ouverte précédentes, introduisons un bouclage reposant sur la passivité. On note 


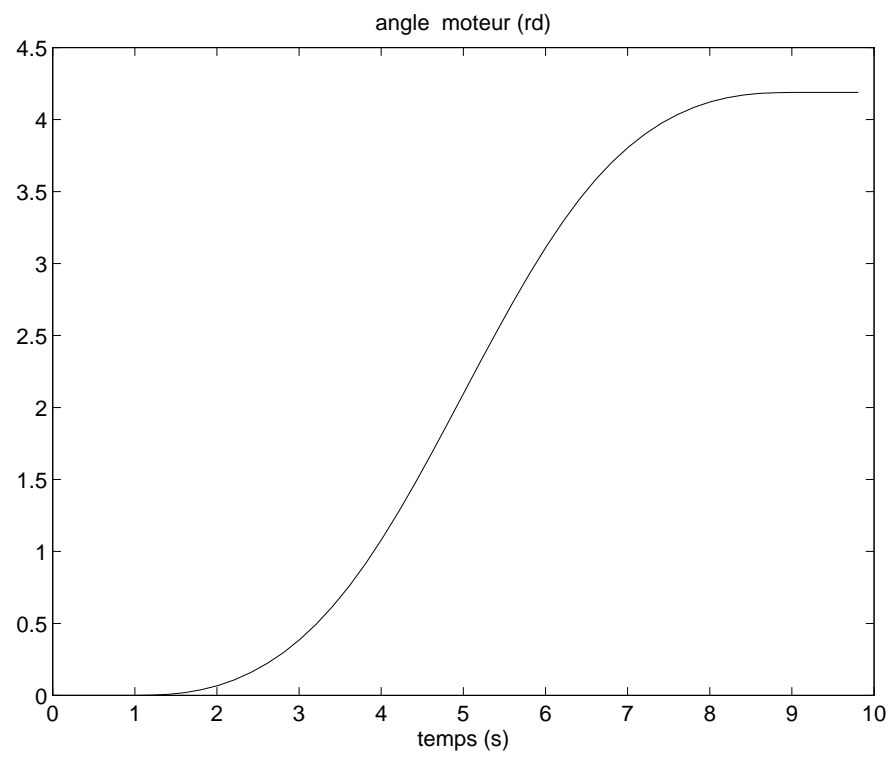

Figure 3. trajectoire de l'angle $\theta(t)$.

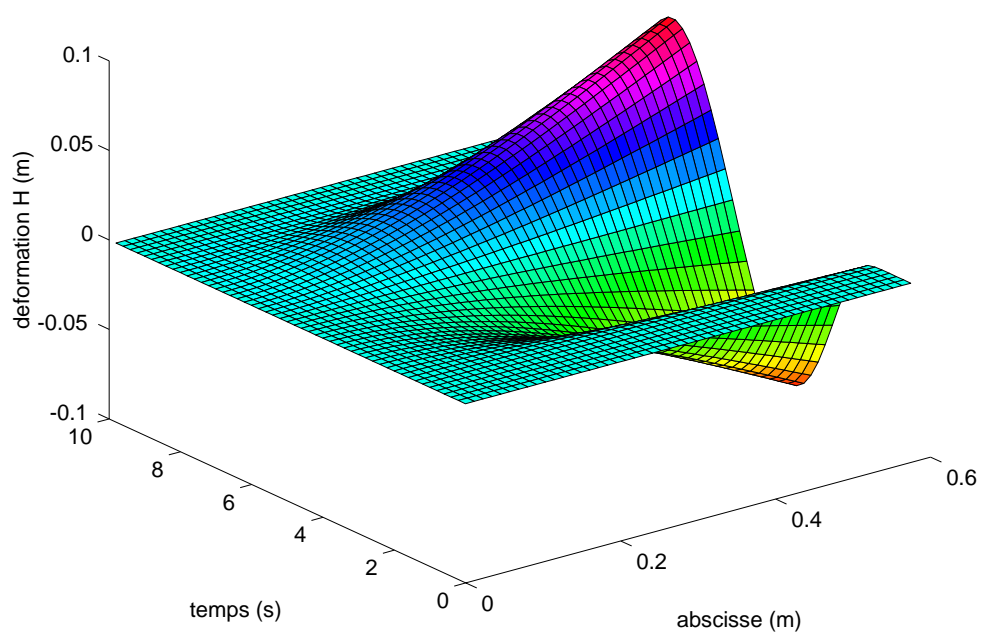

Figure 4. le champ de déformation $w(r, t)$.

$u_{c}(\tau)$ et $w_{c}(x, \tau)$ la trajectoire de consigne pour $u$ et $w$. Ainsi la trajectoire de référence en angle $\theta_{c}$ est donnée par $\theta_{c}(\tau)=\left(u_{c}-k_{2} w_{c}(1, \tau)\right) / k_{1}$. La commande physique étant le couple moteur $\mathcal{C}$ de référence $\mathcal{C}_{c}(\tau)=$ $-E I / R^{2} \frac{\partial^{2} w_{c}}{\partial x^{2}}(0, \tau)$, un bouclage élémentaire du type ressort et friction de rappel à la trajectoire de référence, utilisant uniquement l'angle $\theta$ et sa vitesse $\frac{d \theta}{d \tau}$,

$$
\mathcal{C}=\mathcal{C}_{c}(\tau)-k\left(\theta-\theta_{c}(\tau)\right)-\sigma\left(\frac{d \theta}{d \tau}-\frac{d \theta_{c}}{d \tau}(\tau)\right)
$$

avec $k$ et $\sigma$ deux paramètres positifs, assure le suivi asymptotique de la consigne. 
Remerciements : Les concours financiers du département S.P.I. du C.N.R.S. et du programme H.C.M. de l'Union Européenne (réseau Chemical Process Control - contrat CHRX-CT94-0672) ont permis à l'un des auteurs (J.R.) des séjours au Laboratoire des Signaux et Systèmes du C.N.R.S. et au Centre Automatique et Systèmes de l'École des Mines de Paris.

\section{REFERENCES}

[1] Y. Aoustin, C. Chevallereau, A. Glumineau et C.H. Moog, Experimental results for the end-effector control of a single flexible robotic arm, IEEE Trans. Control Systems Technol., 2, 1994, p. 371-381.

[2] J. Baillieul et M. Levi, Rotational elastic dynamics, Physica D, 27, 1987, p. 43-62.

[3] A. Bensoussan, G. Da Prato, M.C. Delfour et S.K. MitTer, Representation and Control of Infinite Dimensional Systems, t. 1 \& 2, Birkhäuser, Boston, 1992 \& 1993.

[4] F. Bourquin, Contribution à l'analyse numérique et au contrôle des systèmes élastiques couplés, Thèse Habilit., Université P. \& M. Curie, Paris, 1995.

[5] F. Boyer et P. CoIffet, Symbolic modelling of a flexible manipulator via assembling of its generalized Newton Euler model, Mech. Mach. Theory, 31, 1996, p. 45-56.

[6] J. Brewer, J. Bunce et F.V. Vleck, Linear Systems over Commutative Rings, Marcel Dekker, New York, 1986.

[7] D. Buchsbaum et D. Eisenbud, What makes a complex exact?, J. Algebra, 25, 1973, p. 259-268.

[8] C. Castro et E. Zuazua, Analyse spectrale et contrôle d'un système hybride composé de deux poutres connectées par une masse ponctuelle, C.R. Acad. Sci. Paris, I-322, 1996, p. 351-356.

[9] P.-M. Chang et S. Jayasuriya, An evaluation of several controller synthesis methodologies using a rotating flexible beam as a test bed, J. Dynamic Systems Meas. Control, 117, 1995, p. 360-373.

[10] G. Chen, M.C. Delfour, A.M. Krall et G. Payre, Modeling, stabilization, and control of serially connected beams, SIAM J. Control Optimiz., 25, 1987, p. 526-546.

[11] R.F. Curtain et H.J. ZwART, An Introduction to Infinite-Dimensional Linear Systems Theory, Springer, New York, 1995.

[12] P. Destuynder, I. Legrain, L. Castel et N. Richard, Theoretical, numerical and experimental discussion on the use of piezoelectric devices for control-structure interaction, Europ. J. Mecha. A/Solids, 11, 1992, p. 181-213.

[13] V. Ditkine et A. Proudnikov, Transformations intégrales et calcul opérationnel (traduit du russe), Mir, Moscou, 1978.

[14] D. EISEnBUD, Commutative Algebra with a View Toward Algebraic Geometry, Springer, New York, 1995.

[15] A. EL J $\mathrm{AI}$ et A.J. PRITChARD, Capteurs et actionneurs dans l'analyse des systèmes distribués, Masson, Paris, 1986.

[16] M. FLIEss, Some basic structural properties of generalized linear systems, Systems Control. Lett., 15, 1990, p. 391-396.

[17] M. FliEss, A remark on Willems' trajectory characterization of linear controllability, Systems Control Lett., 19, 1992, p. 43-45.

[18] M. FLIEss, Reversible linear and nonlinear discrete-time dynamics, IEEE Trans. Automat. Control, 37, 1992, p. 1144-1153.

[19] M. FliEss, Une interprétation algébrique de la transformation de Laplace et des matrices de transfert, Linear Algebra Appl., 203-204, 1994, p. 429-442.

[20] M. Fliess et H. Bourlès, Discussing some examples of linear system interconnections, System Control Lett., 27, 1996, p. 1-7.

[21] M. Fliess et R. Hotzel, Sur les systèmes linéaires à dérivation non entière, C.R. Acad. Sci. Paris, II-324, 1997.

[22] M. Fliess, J. Lévine, P. Martin et P. Rouchon, Flatness and defect of nonlinear systems: introductory theory and applications, Internat. J. Control, 61, 1995, p. $1327-1361$.

[23] M. Fliess et H. Mounier, Quelques propriétés structurelles des systèmes linéaires à retards constants, C.R. Acad. Sci. Paris, I-319, 1994, p. 289-294. 
[24] M. Fliess et H. Mounier, Interpretation and comparison of various types of delay system controllabilities, Proc. IFAC Conf. System Struct. Control, Nantes, 1995, p. 330-335.

[25] M. Fliess, H. Mounier, P. Rouchon et J. Rudolph, Controllability and motion planning for linear delay systems with an application to a flexible rod, Proc. $34^{\text {th }}$ Conf. Decision Control, New Orleans, 1995, p. 2046-2051.

[26] I.M. Guelfand ET G.E. Chilov, Les distributions ; $t$. 2 : espaces fondamentaux (traduit du russe), Dunod, Paris, 1964.

[27] V. KomorNik, Exact Controllability and Stabilization, Wiley, Chichester \& Masson, Paris, 1994.

[28] J.E. LAgnese et J.-L. Lions, Modelling Analysis and Control of Thin Plates, Masson, Paris, 1988.

[29] S. LAng, Algebra, $3^{e}$ éd., Addison-Wesley, Reading, MA, 1993.

[30] H. LAOUsy, C.Z. Xu et G. SALLET, Boundary feedback stabilization of a rotating body-beam system, IEEE Trans. Automat. Control, 41, 1996.

[31] J.-L. Lions, Contrôle optimal des systèmes gouvernés par des équations aux dérivées partielles, Dunod, Paris, 1968.

[32] J.-L. LIONs, Contrôlabilité exacte, perturbations et stabilisation des systèmes distribués, t. 1 \& 2, Masson, Paris, 1988.

[33] J. Mikusiński, Operational Calculus, t. 1, Pergamon, Oxford \& PWN, Varsovie, 1983.

[34] J. Mikusiński et T.K. Bofhme, Operational Calculus, t. 2, Pergamon, Oxford \& PWN, Varsovie, 1987.

[35] O. MorgüL, Dynamic boundary control of a Euler-Bernoulli beam, IEEE Trans. Automat. Control, 37, 1992, p. 639-652.

[36] O. MorgüL, Control and stabilization off a rotating flexible structure, Automatica, 30, 1994, p. 351-356.

[37] H. MouniER, An algebraic interpretation of the spectral controllability of a linear delay system, Proc. $3^{\text {rd }}$ Europ. Control Conf., Rome, 1995, p. 3325-3329.

[38] H. Mounier, Propriétés structurelles des systèmes linéaires à retards : aspects théoriques et pratiques, Thèse, Université Paris-Sud, Orsay, 1995.

[39] H. MounIER, Algebraic interpretations of the spectral controllability of a linear delay system, Forum Math., à paraître.

[40] H. Mounier, J. Rudolph, M. Petitot et M. Fliess, A flexible rod as a linear delay system, Proc. $3^{\text {rd }}$ Europ. Control Conf., Rome, 1995, p. 3676-3681.

[41] A. Oustaloup, La dérivation non entière, Hermès, Paris, 1995.

[42] D. Quillen, Projective modules over polynomial rings, Inventiones Math., 36, 1976, p. $167-171$

[43] J.-P. Ramis, Dévissage Gevrey, Astérisque, 59-60, 1979, p. 173-204.

[44] J.-P. Ramis, Séries divergentes et théories asymptotiques, Soc. Math. France, Marseille, 1993.

[45] J. Rotman, An Introduction to Homological Algebra, Academic Press, Orlando, 1979.

[46] L.H. Rowen, Ring Theory, Academic Press, Boston, 1991.

[47] J. Rudolph, Duality in Time-Varying Linear Systems: A Module Theoretic Approach, Linear Algebra Applications, à paraître, 1996.

[48] P. SchapIRA, Microdifferential Systems in the Complex Domain, Springer, Berlin, 1985.

[49] A.A. Suslin, Projectives modules over a polynomial ring are free (en russe), Dokl. Akad. Nauk. SSSR, 229, 1976, p. 1063-1066 (traduction anglaise: Soviet. Math. Dokl. 17, 1976, p. 1160-1164).

[50] M. TuCSNAK, Regularity and exact controllability for a beam with piezoelectric actuator, SIAM J. Control Optimiz., 34, 1996, p. 922-930.

[51] J.C. Willems, Paradigms and puzzles in the theory of dynamical systems, IEEE Trans. Automat. Control, 36, 1991, p. 259-294.

[52] C.Z. Xu et J. BAIllieul, Stabilizability and stabilization of a rotating body-beam system with a torque control, IEEE Trans. Automat. Control, 38, 1993, p. 1754-1765.

[53] K. YosidA, Operational Calculus, Springer, New York, 1984. 
Laboratoire des Signaux et Systèmes, C.N.R.S.-Supélec, Plateau de MouLon, 91192 Gif-Sur-Yvette, France, fliess@lss.supelec.fr

Département Automatique et Productique, École des Mines de Nantes, 4, rue Alfred Kastler, 44072 Nantes Cedex 03, France, mounier@1ss.supelec.fr

Centre Automatique et Systèmes, École des Mines de Paris, 60, Bld. SaintMichel, 75272 Paris Cedex 06, France, rouchon@cas.ensmp.fr

Lehrstuhl FüER Regelungs und Steuerungstheorie, Technische Universität Dresden, Mommsenstr. 13, D-01062 Dresden, Allemagne, rudolphoerss11. et.tu-dresden. de 